\title{
Choice of the electronic basis for field-induced surface hopping
}

\author{
Murali Krishna Ganesa Subramanian $\mathbb{0}^{1,2}$ Robin Santra ${ }^{10},{ }^{1,2,3}$ and Ralph Welsch ${ }^{1,3}$ \\ ${ }^{1}$ Center for Free-Electron Laser Science, DESY, Notkestrasse 85, 22607 Hamburg, Germany \\ ${ }^{2}$ Department of Physics, Universität Hamburg, Jungiusstrasse 9, 20355 Hamburg, Germany \\ ${ }^{3}$ The Hamburg Centre for Ultrafast Imaging, Luruper Chaussee 149, 22761 Hamburg, Germany
}

(Received 16 January 2020; accepted 2 June 2020; published 16 July 2020)

\begin{abstract}
A method combining an Ehrenfest-like approach, which minimizes the number of surface hops, with Tully's fewest switches surface hopping is proposed for the description of nonadiabatic molecular dynamics in the presence of an external electromagnetic field. When two states are strongly coupled by the applied light field, an Ehrenfest-like approach is employed, which allows the system to be in a coherent superposition. Tully's fewest switches surface hopping (FSSH) is used for the weak- and no-coupling regimes to improve the asymptotic behavior of the method. The decision of which approach to employ at a given time is made based on a simple analysis of Rabi oscillations in a two-state model. The method is tested for two exactly solvable model systems, i.e., a stimulated emission scenario in a pulsed laser field and a photoexcitation scenario in a cw laser field. Position and momentum densities of the nuclei compare well with exact quantum dynamics simulations and improve on both a pure Ehrenfest and a pure FSSH approach. The method is efficient and easily implemented.
\end{abstract}

DOI: 10.1103/PhysRevA.102.013107

\section{INTRODUCTION}

Key phenomena in molecular processes such as charge transfer and transport [1-5], isomerization [6,7], photodeactivation $[8,9]$, or singlet fission [10] can be understood by investigating the dynamics on an atomic scale. In many of these processes, the Born-Oppenheimer approximation breaks down and we have to treat the coupled motion of electrons and nuclei. While exact, full-dimensional quantum dynamics approaches can readily simulate this, the computational effort of these methods scales exponentially with increasing system size. Therefore, mixed quantum classical descriptions, i.e., approaches employing a classical description of the nuclei and a quantum description of the electrons, have been developed. These methods include the Ehrenfest or mean-field approach $[11,12]$ and Tully's fewest switches surface hopping (FSSH) approach [13-15].

In Ehrenfest dynamics, the nuclei evolve classically due to a force from a superposition of electronic eigenstates. However, due to the averaged potential, Ehrenfest dynamics fails to correctly describe processes with multiple reaction paths or processes that end up in distinct states that are well described by a single potential energy surface (PES). These processes can be better described by the popular FSSH approach. In FSSH, each classical trajectory evolves on a single PES and stochastically hops between the different PESs. The transition probabilities depend on the electronic population and on the nonadiabatic coupling (NAC) vector. For every hop, the velocities are scaled to conserve energy. By employing this energy-conservation procedure, the FSSH algorithm can approximately reproduce the Boltzmann distribution of quantum states. It allows for the FSSH method to investigate the relaxation of quantum states to thermal equilibrium [16], which is not possible in the Ehrenfest approach [16,17]. FSSH is an appealing approach due to its conceptual simplicity and numerical efficiency, while being reasonably accurate. However, FSSH has several limitations such as missing nuclear quantum effects and quantum interference, lack of proper decoherence, and representation dependence [18]. In recent years, there has been a considerable theoretical advancement to overcome such limitations. Some of the recently developed FSSH-like algorithms include ring polymer surface hopping [19-24], global flux surface hopping (GFSH) [25], Liouville space FSSH [26], Liouville space GFSH [27], decoherenceinduced surface hopping [28], and many others [29-38].

Further developments of the FSSH algorithm have focused on incorporating an external electric field to investigate lightinduced nonadiabatic dynamics [39-42]. In field-induced FSSH, the PESs are optically coupled by the transition dipole moment $\mu$. The basic states that are then employed in the FSSH algorithm can either be the field-free, adiabatic BornOppenheimer surfaces (BO-SH) [39,43-50] or instantaneous Born-Oppenheimer surfaces that are eigenstates of the electronic Hamiltonian including the electric field at time $t$ (IBO$\mathrm{SH}$ ) [51-56]. However, it has recently been shown that for photoexcitation and stimulated emission processes in an exactly solvable $\mathrm{H}_{2}{ }^{+}$model, these approaches suffer from an unphysically high average number of hops per trajectory, which lead to nonphysical results compared to full quantum simulations [57]. Another FSSH variant employing Floquet surfaces within the FSSH scheme, i.e., Floquet surface hopping (F-SH) [40,57], was found to reduce the average number of hops and, consequently, better reproduce the exact results. Yet, the Floquet picture is strictly exact only for time-periodic cw laser fields. In the present work, we propose an improved version of field-induced BO-SH, which we term optimized BO-SH (o-BO-SH), in which the number of hops per trajectory is minimized. To this end, the method employs an Ehrenfest-like approach in the strongly coupled region and regular BO-SH elsewhere. A simple analysis of Rabi oscillations in a two-state 
model allows for the distinction of these two situations. The method is compared to exact quantum calculations as well as other surface hopping variants discussed in Ref. [57]. Similar ideas have been proposed for nonadiabatic dynamics without an external field [58-62], which will be discussed in Sec. IV.

The paper is organized as follows: In Sec. II, the model system employed in this work is introduced, the basic fieldinduced FSSH approach is reviewed, and the method proposed in this work is discussed in detail. Section III presents results on a model system and comparison to other works. Section IV gives a general discussion of the presented method and puts it into perspective, while Sec. V presents some conclusions.

\section{THEORETICAL BACKGROUND}

\section{A. Model system and potential energy surfaces}

In order to facilitate a systematic comparison, we follow Ref. [57] and employ a soft-core Hamiltonian given as

$$
\begin{aligned}
H= & -\frac{\Delta_{R}}{2 M}-\frac{\Delta_{r}}{2}+\frac{1}{R+0.03}-\mu E(t) \\
& -\frac{1}{\sqrt{(r-R / 2)^{2}+1}}-\frac{1}{\sqrt{(r+R / 2)^{2}+1}},
\end{aligned}
$$

where $M$ is the reduced nuclear mass, $R$ and $r$ are the nuclear and electronic coordinates, respectively, and $\mu$ is the transition dipole moment operator. The $\mathrm{BO}$ surfaces $E_{0 / 1}^{\mathrm{BO}}(R)$ are calculated by diagonalizing the field-free Hamiltonian for fixed nuclear coordinates [57],

$$
\begin{aligned}
H^{\mathrm{BO}}(R)= & -\frac{\Delta_{r}}{2}+\frac{1}{R+0.03}-\frac{1}{\sqrt{(r-R / 2)^{2}+1}} \\
& -\frac{1}{\sqrt{(r+R / 2)^{2}+1}} .
\end{aligned}
$$

The IBO Hamiltonian includes the electric-field interaction $\mu E(t)$ and reads

$$
H^{\mathrm{IBO}}(R, t)=H^{\mathrm{BO}}(R)-\mu E(t),
$$

with the corresponding IBO energies $E_{0 / 1}^{\mathrm{IBO}}(R, t)$ given by [57]

$$
\begin{aligned}
E_{0 / 1}^{\mathrm{IBO}}(R, t) & =E_{0 / 1}^{\mathrm{BO}}(R) \cos ^{2} \theta(R, t)+E_{1 / 0}^{\mathrm{BO}}(R) \sin ^{2} \theta(R, t) \\
& \pm \mu_{01}^{\mathrm{BO}}(R) E(t) \sin 2 \theta(R, t),
\end{aligned}
$$

where the mixing parameter $\theta(R, t)$ is given by [57]

$$
\theta(R, t)=\frac{1}{2} \arctan \frac{2 \mu_{01}^{\mathrm{BO}}(R) E(t)}{E_{0}^{\mathrm{BO}}(R)-E_{1}^{\mathrm{BO}}(R)} .
$$

In this work, we have considered two cases of molecular dissociation following Ref. [57]: a $\mathrm{Na}_{2}{ }^{+}$-like molecule $(M=23 \times 918$ a.u. $)$ undergoing stimulated emission due to a Gaussian-shaped laser pulse with intensity $I=3 \times$ $10^{12} \mathrm{~W} / \mathrm{cm}^{2}$ (see Sec. III A) and an $\mathrm{H}_{2}{ }^{+}$-like molecule $(M=$ 918 a.u.) undergoing photon absorption due to a $\mathrm{cw}$ laser with intensity $I=1 \times 10^{13} \mathrm{~W} / \mathrm{cm}^{2}$ (see Sec. III B). In the latter case, the initial trajectories were given an additional momentum of -2.5 a.u. The photon energy of the electromagnetic field is $\hbar \omega=0.2$ a.u., which resonantly couples the lowest

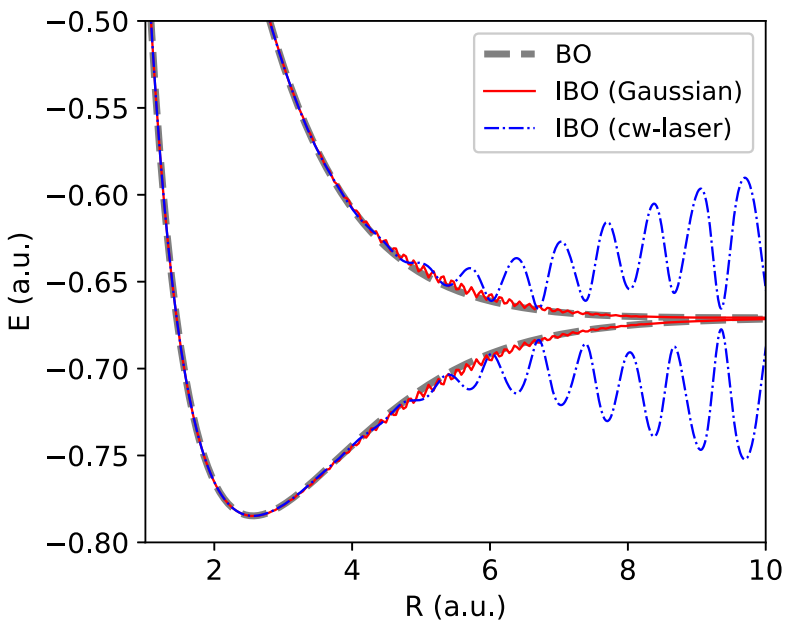

FIG. 1. The two lowest BO and IBO PESs [for both cases: stimulated emission (curve with smaller oscillations) and photon absorption (curve with bigger oscillations)], with the equilibrium distance, $R_{\mathrm{eq}} \approx 2.3$ a.u. The IBO surfaces were obtained by evolving a single classical trajectory, starting on the upper PES at $R=$ 0.85 a.u. with zero initial momentum and employing the electric fields and reduced nuclear masses described in Sec. II A. As a consequence, the IBO PESs do not correspond to any single timedifferent points in space are calculated at different times.

two PESs at around $R=3.5$ a.u. The two PESs are optically coupled via the dipole moment, which for the model system is approximated as $\mu_{k j}=\delta_{k j} R / 2$. Figure 1 shows the lowest two BO and IBO PESs (for both cases described above).

\section{B. Fewest switches surface hopping}

A central component of the method proposed in this work is Tully's FSSH approach and the implementation largely follows Ref. [63]. In FSSH, the electrons are treated quantum mechanically, while the nuclei are treated classically, propagating along a trajectory $R(t)$. The classical trajectories evolve on a single PES and can switch between the electronic states based on a hopping probability. In the model cases employed here, the PESs are coupled only through the transition dipole moment $\mu_{k j}$ and, hence, we set the nonadiabatic-coupling vector $d_{k j}=0$ throughout the simulations. We will focus on one-dimensional systems here, but the approach can be easily generalized to many degrees of freedom. We employ different field-induced FSSH variants. In BO-SH, we solve the timedependent electronic Schrödinger equation (TDSE) along the nuclear trajectories employing the $\mathrm{BO}$ surfaces,

$$
i \dot{c}_{k}(t)=\sum_{j} c_{j}(t)\left[V_{k j}^{\mathrm{BO}}-E(t) \mu_{k j}\right],
$$

where the $c_{k}$ 's are the expansion coefficients of the electronic wave function and the $V_{k j}^{\mathrm{BO}}$ are the matrix elements of the field-free electronic Hamiltonian in the adiabatic representation, i.e., they are zero for $k \neq j$, and for $k=j$ they are the eigenvalues of the field-free Hamiltonian for fixed nuclei coordinates [i.e., $\left.E_{0 / 1}^{\mathrm{BO}}(R)\right]$. The rate of change of the electronic 
population is given by

$$
\dot{\rho}_{j j}(t)=\sum_{k \neq j} 2 \operatorname{Re}\left[i \rho_{j k}(t) E(t) \mu_{k j}\right] .
$$

The probability of hopping from state $j$ to state $k, P_{j \rightarrow k}$, is obtained as [63]

$$
P_{j \rightarrow k}(t)=\frac{-2 \operatorname{Re}\left[i \rho_{j k} E(t) \mu_{k j}\right] \Delta t}{\rho_{j j}} .
$$

In case of IBO-SH, we use the IBO surfaces, $E_{0 / 1}^{\mathrm{IBO}}(R)$, and the time evolution of the expansion coefficients is derived using the Hellmann-Feynman theorem:

$$
i \dot{c}_{k}(t)=\sum_{j} c_{j}(t)\left[V_{k j}^{\mathrm{IBO}}-\frac{i \dot{E}(t) \mu_{k j}}{\Delta E_{j k}^{\mathrm{IBO}}}\right],
$$

where $\Delta E_{j k}^{\mathrm{IBO}}=E_{j}^{\mathrm{IBO}}-E_{k}^{\mathrm{IBO}}$. The rate of change of the electronic population is given by

$$
\dot{\rho}_{j j}(t)=-\sum_{k \neq j} 2 \operatorname{Re}\left[\frac{\rho_{j k} \dot{E}(t) \mu_{k j}}{\Delta E_{j k}^{\mathrm{IBO}}}\right],
$$

and the probability of hopping from state $j$ to state $k$ is given as

$$
P_{j \rightarrow k}(t)=2 \operatorname{Re}\left[\frac{\rho_{j k}(t) \dot{E}(t) \mu_{k j}}{\Delta E_{j k}^{\mathrm{IBO}}}\right] \frac{\Delta t}{\rho_{j j}(t)} .
$$

We assume that the energy required for any hop is provided by the field and thus do not perform any velocity adjustments after a hop occurs.

\section{Optimized BO-SH}

In this work, we propose a modified FSSH approach that we term the "optimized" BO-SH (o-BO-SH) method. It consists of two parts described in detail below. The first part aims at reducing the number of hops. To this end, we employ a time-dependent transformation of the electronic basis. For the two-state model investigated here, this part is equivalent to an Ehrenfest approach. The second part consists of a criterion for when to employ the optimization and when to employ standard BO-SH. This part is based on the description of Rabi oscillations.

\section{Optimization}

In order to reduce the number of field-induced hops, the underlying electronic basis is transformed. The transformation matrix at each time step is obtained by minimizing the instantaneous hopping probability. For a two-state case, the transformation matrix reads

$$
U(\theta, \phi)=\left[\begin{array}{lc}
\cos \theta & \sin \theta e^{i \phi} \\
-\sin \theta e^{-i \phi} & \cos \theta
\end{array}\right],
$$

where $\theta$ and $\phi$ are parameters that are optimized at each time step and thus carry the time dependence. We note that there is no need to optimize the global phase of the electronic wave packet. Therefore, the parametrization employed in Eq. (12) captures all relevant $\mathrm{SU}(2)$ transformation matrices. Parametrizations for $\mathrm{SU}(n)$ matrices with $n>2$ (i.e., for electronic subspaces consisting of more than two states) are available but are less straightforward [64].

The corresponding time-dependent Schrödinger equation in the new basis (labeled with a prime) reads

$$
\begin{aligned}
i{\dot{c^{\prime}}}_{k}(t)= & \sum_{j} c_{j}^{\prime}(t)\left[\sum_{a} U_{k a}(\theta, \phi) V_{a a}^{\mathrm{BO}} U_{a j}^{\dagger}(\theta, \phi)\right. \\
& -\sum_{a, b} U_{k a}(\theta, \phi) E(t) \mu_{a b} U_{b j}^{\dagger}(\theta, \phi) \\
& \left.-i \sum_{a} U_{k a}(\theta, \phi) \dot{U}_{a j}^{\dagger}(\theta, \phi)\right]
\end{aligned}
$$

and the probability of hopping from a state $j$ to state $k$ is given by

$$
P_{j \rightarrow k}(t)=\frac{-2 \operatorname{Re}\left\{\left[-i \sum_{a} U_{k a} V_{a a}^{\mathrm{BO}} U_{a j}^{\dagger}+i \sum_{a, b} U_{k a} E(t) \mu_{a b} U_{b j}^{\dagger}+\sum_{a} U_{k a} \dot{U}_{a j}^{\dagger}\right] \rho_{k j}^{\prime}\right\} \Delta t}{\rho_{j j}^{\prime}} .
$$

Therefore, $P_{j \rightarrow k}$ is a function of $\theta$ and $\phi$. Please note that $j$ and $k$ in Eq. (14) do not refer to BO PESs but to "optimized" PESs defined below. To reduce the number of hops, we minimize $P_{j \rightarrow k}$ with respect to $\theta$ and $\phi$, i.e., $\min _{\theta, \phi} P_{j \rightarrow k}(\theta, \phi)=$ $P_{j \rightarrow k}\left(\theta_{\text {opt }}, \phi_{\text {opt }}\right)$ and obtain optimal values $\theta_{\text {opt }}$ and $\phi_{\text {opt }}$ at each time step. We perform the minimization using the function minimize from the Python library SciPy [65]. The temporal derivative of $U$, required for evaluating the hopping probability in Eq. (14), can be expressed in terms of $\dot{\theta}$ and $\dot{\phi}$, which we compute employing first-order finite differencing. This approach is numerically stable as long as the time step is small enough.

We carry out the standard FSSH algorithm with the new set of optimized PESs $V^{o}=U\left(\theta_{\text {opt }}, \phi_{\text {opt }}\right) V^{\mathrm{BO}} U^{\dagger}\left(\theta_{\text {opt }}, \phi_{\text {opt }}\right)$. Each classical trajectory then evolves on the optimized PES $V_{j}^{o}$, where $j$ labels the current active electronic state of the trajectory just as in FSSH. Gradients for solving Newton's equations of motion are obtained as approximate derivatives of the optimized PES, i.e., as

$$
\frac{\partial}{\partial R} V_{j}^{o}=\sum_{a} U_{j a}\left(\theta_{\mathrm{opt}}, \phi_{\mathrm{opt}}\right)\left(\frac{\partial}{\partial R} V_{a a}^{\mathrm{BO}}\right) U_{a j}^{\dagger}\left(\theta_{\mathrm{opt}}, \phi_{\mathrm{opt}}\right) .
$$

\section{Criterion for employing the optimization}

In order to reduce the field-induced hops, the optimization procedure is carried out when there is strong optical coupling through the transition dipole moment $\mu$. However, as mentioned above, we find that for the cases investigated here, the optimization procedure described above results in an approach very close to the Ehrenfest approach. However, once the coupling is weak, the system will stay in the optimized, averaged state, which can lead to nonphysical behavior if the 


$$
t=40.00 \text { fs }
$$
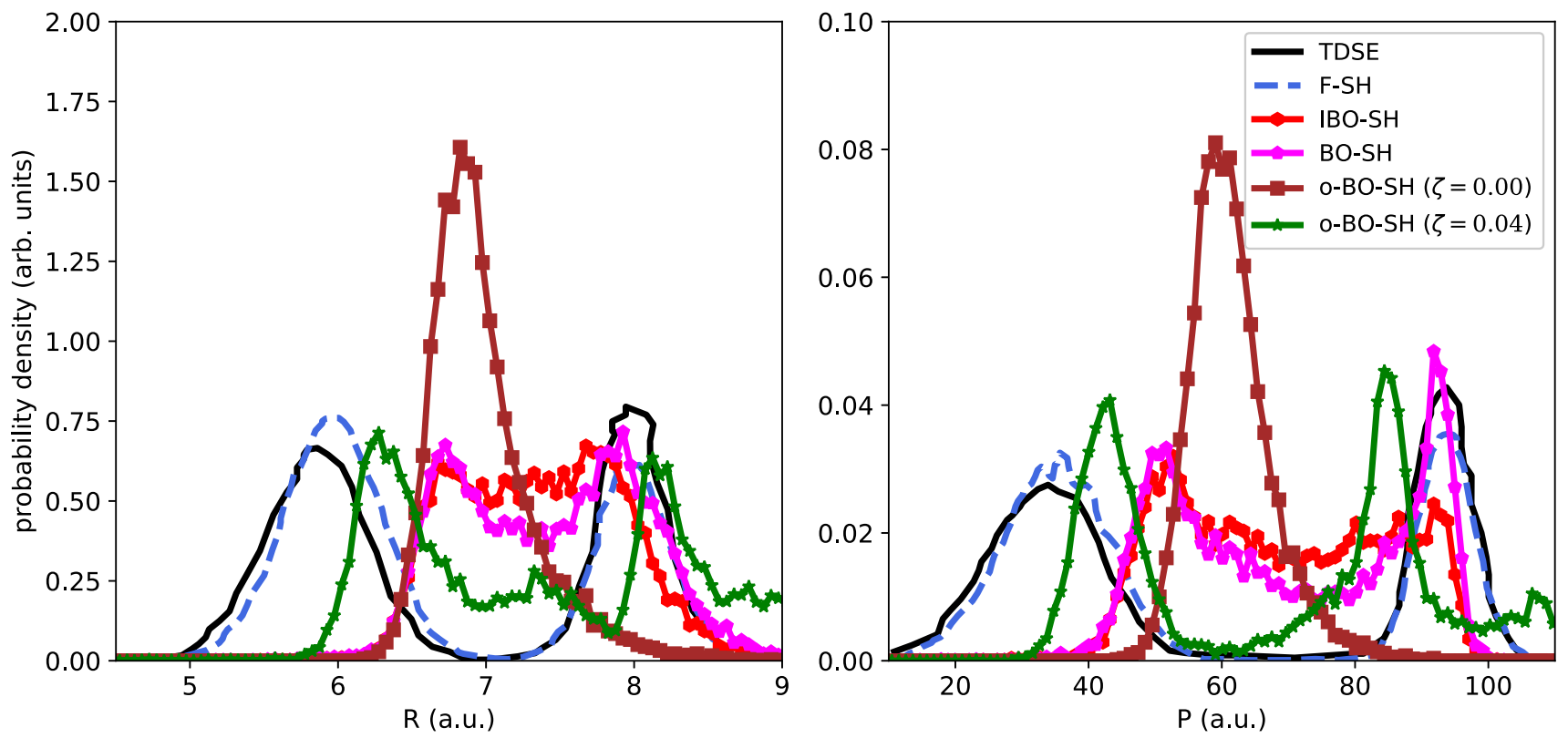

FIG. 2. The nuclear densities for scenario 1 at $t=40 \mathrm{fs}$, in position (left panel) and momentum (right panel) space, obtained from BO-SH, IBO-SH, and o-BO-SH for $\zeta=0.00$ and o-BO-SH for $\zeta=0.04$, in the presence of a Gaussian pulse, in comparison to TDSE and F-SH from Ref. [57].

two states show distinct gradients, similar to the problems arising in Ehrenfest dynamics. Thus, once the strength of the optical coupling is small, one should revert back to employing the normal BO PESs. To determine if the coupling is small, we employ a resonantly coupled two-level model exhibiting Rabi oscillations. The probability for switching the state in this model is given as

$$
P_{\text {Rabi }}=\frac{\left|\epsilon(t) \mu_{01}\right|^{2}}{\left(\omega-\Delta E_{01}^{\mathrm{BO}}\right)^{2}+\left|E(t) \mu_{01}\right|^{2}},
$$

where $\omega$ and $\epsilon(t)$ are the photon energy and the envelope associated with the electric field $E(t)=\epsilon(t) \cos (\omega t)$, and $\Delta E_{01}^{\mathrm{BO}}=E_{0}^{\mathrm{BO}}-E_{1}^{\mathrm{BO}}$ is the energy difference of the two $\mathrm{BO}$ states. Therefore, once the switching probability is above a threshold value $\zeta$, we employ the optimization algorithm described above and, if it is below that threshold value, we switch back to the BO PESs. To this end, we calculate the absolute square of the expansion coefficients of the current electronic state with respect to the $k$-th $\mathrm{BO}$ state, which gives the probability to switch to the $k$-th BO PES. A Monte Carlo strategy similar to the standard FSSH approach is employed to sample these probabilities.

\section{RESULTS}

The model systems employed in this work are described in Sec. II A. We consider two cases of molecular dissociation: A $\mathrm{Na}_{2}{ }^{+}$-like molecule undergoing stimulated emission due to a Gaussian pulse (see Sec. III A) and an $\mathrm{H}_{2}{ }^{+}$-like molecule undergoing photon absorption due to a cw laser (see Sec. III B). Throughout this work, we employ 10000 classical trajectories sampled using Wigner sampling. Trajectories are propa- gated using the Velocity-Verlet approach with a time step of 0.002 a.u.

\section{A. Scenario 1: Stimulated emission in Gaussian pulse}

First we discuss stimulated emission from an electronically excited $\mathrm{Na}_{2}{ }^{+}$-like molecule $(M=23 \times 918$ a.u. $)$ by a Gaussian pulse with $7 \mathrm{fs}$ duration (full width at half maximum of the intensity), wavelength of $\lambda=225 \mathrm{~nm}$, and peak intensity of $I=3 \times 10^{12} \mathrm{~W} / \mathrm{cm}^{2}$.

Figure 2 shows the nuclear position and momentum densities at $t=40 \mathrm{fs}$, obtained from BO-SH, IBO-SH, and oBO-SH in comparison with results from Ref. [57] employing F-SH and exact TDSE calculations. Here, position means internuclear distance, and momentum refers to the relative momentum of the two nuclei. The latter is positive when the two nuclei are moving away from each other; it is negative when they are approaching each other. The exact momentum density in Fig. 2(b) shows that the wave packet splits into a fast moving part (mean momentum $P \approx 94$ a.u. at $t=$ $40 \mathrm{fs}$ ) and a slow moving part (mean momentum $P \approx 34$ a.u. at $t=40 \mathrm{fs}$ ). The fast moving part is on the ground electronic state and shows a mean position of $R \approx 5.9$ a.u., while the slow moving part is located on the excited electronic state and shows a mean position of $R \approx 7.9$ a.u.

The F-SH simulations from Ref. [57] show a similar behavior. However, both the BO-SH and IBO-SH approaches show a less distinct splitting in the densities. As discussed in Ref. [57], this failure can be attributed to the high number of hops per trajectory in these two approaches compared to F-SH (see also Table I). The optimization method introduced in this work reduces the number of hops. Yet, the pure optimization method (i.e., $\zeta=0$ ), which reduces the number of hops to 0 , 
TABLE I. The average number of hops per trajectory in different FSSH methods for scenarios 1 and 2. The numbers for F-SH are taken from Ref. [57] (note the interchange of scenarios 1 and 2).

\begin{tabular}{lcc}
\hline \hline Method & Scenario 1 & Scenario 2 \\
\hline F-SH & 0.6 & 0.01 \\
BO-SH & 1.45 & 3.16 \\
IBO-SH & 2.15 & 42.3 \\
o-BO-SH $(\zeta=0.00)$ & 0.00 & 0.00 \\
o-BO-SH $(\zeta=0.04)$ & 0.98 & 0.22 \\
o-BO-SH $(\zeta=0.10)$ & 1.13 & \\
o-BO-SH $(\zeta=0.50)$ & 2.21 & \\
\hline \hline
\end{tabular}

shows no splitting at all due to the Ehrenfest-like character of that approach. If a larger value of $\zeta$ is employed, $\zeta=0.04$ in this case, the trajectories are put onto the correct BO PES after they passed the coupling region and a distinct splitting of the distribution is found, which is closer to the exact values than pure BO- or IBO-SH.

The choice of an appropriate value for $\zeta$ is discussed next. It is clear that a too low value of $\zeta$ results in an Ehrenfest-like approach, which does not give reasonable results here, while a too high value of $\zeta$ results in a pure BO-SH approach, which is also unreasonable for the strongly driven systems discussed here. The observation in different tests is that while the coupling is strong, the optimization strategy performs well, and where the coupling is weak, the FSSH approach performs better. By examining Fig. 3, which shows the spatial dependence of the Rabi switching probability given in Eq. (15) for the current scenario, we can decide on a reasonable range for the $\zeta$ parameter. It can be seen that the switching probability peaks at the geometry where the frequency of the external field matches the energy difference of the two electronic states and that it falls off very quickly away from that point. To include

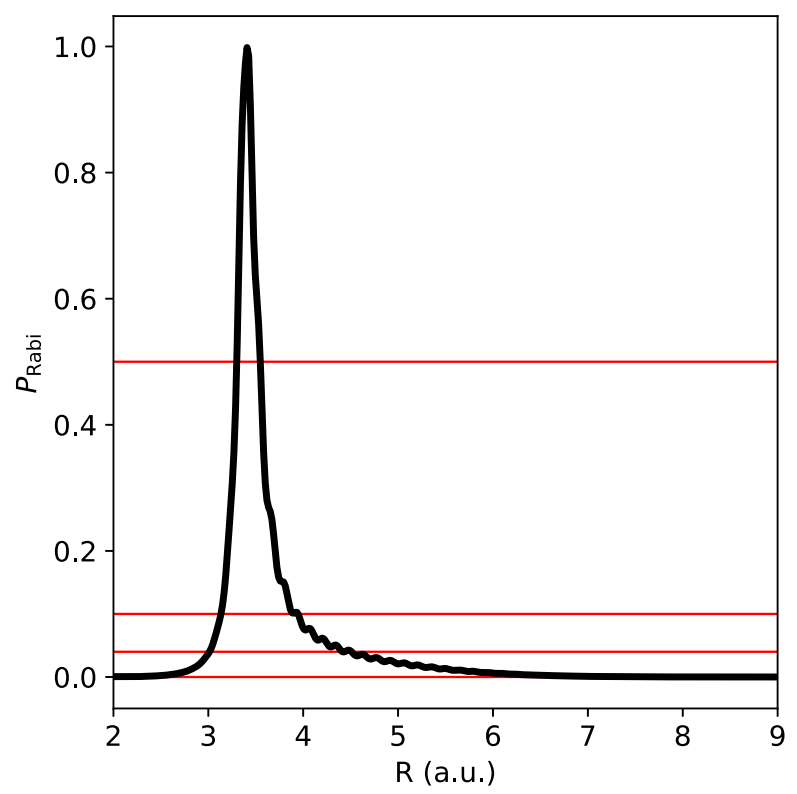

FIG. 3. Rabi switching probability, $P_{\text {Rabi }}$, as a function of position $R$. the strong-coupling regime in the optimization algorithm, one therefore needs to choose a small, but nonzero value of $\zeta$.

To gain better insight into the optimal value as well as the sensitivity of our results with respect to $\zeta$, we performed simulations with $\zeta \in\{0,0.04,0.1,0.5\}$. These values are also indicated as horizontal lines in Fig. 3. In Fig. 4, the resulting nuclear position and momentum densities for simulations with the different values of $\zeta$ are compared. At $t=10 \mathrm{fs}$, before the system enters the interaction region, all densities are equal. However, once the two states are coupled by the external laser field (e.g., around $t=15 \mathrm{fs}$ ), one can see the momentum density splitting up significantly for a high value of $\zeta$ (yellow and cyan line in Fig. 4). This is due to the high- $\zeta$ value forcing the system into one or the other BO PES while there is still significant coupling of the two states. As seen before, a value of $\zeta=0$ results in no splitting of the density and an averaged position and momentum distribution, which is nonphysical.

The effect discussed above can be seen even better in Fig. 5, which shows the momentum density as a function of the $\theta$ optimization parameter at selected times. At $t=15 \mathrm{fs}$ and a high value of $\zeta$ (second row, left column of Fig. 5), one can see that most of the trajectories are located either on the upper or lower BO PES, i.e., $\theta$ is either 0 or $\frac{\pi}{2}$. For $\zeta=0$, one can see that there is no splitting and the systems ends up in an average state, i.e., the values of $\theta$ that are reached are always below $\frac{\pi}{2}$ and above 0 , with an average close to $\frac{\pi}{4}$. Furthermore, over the full time, the distribution in $\theta$ is continuous and relatively compact.

\section{B. Scenario 2: Photon absorption in cw laser}

In this scenario, an $\mathrm{H}_{2}{ }^{+}$-like molecule $(M=918$ a.u.) initially in its ground electronic state, but with an additional momentum of -2.5 a.u., is considered. The system evolves in a cw laser with $I=10^{13} \mathrm{~W} / \mathrm{cm}^{2}$, switched on with a $\sin ^{2}$-shaped ramp (given in Ref. [57]).

Figure 6 shows the nuclear position and momentum densities at $t=25$ fs obtained from BO-SH, IBO-SH, and o$\mathrm{BO}-\mathrm{SH}$ (for $\zeta=0.0$ and 0.04 ) in comparison to calculations employing F-SH and exact TDSE simulations from Ref. [57]. A split in the wave packet is observed in the exact simulation, with one part staying in the ground electronic state and around the equilibrium position and a second part dissociating in the excited electronic state. Again, F-SH gives reasonably good results compared to the exact results. In contrast, both $\mathrm{BO}-\mathrm{SH}$ and IBO-SH underestimate the dissociation probability and give a too small momentum for the dissociating part at $t=$ $25 \mathrm{fs}$. This can be traced back to the high number of hops in BO- and IBO-SH (see Table I).

For this scenario, the o-BO-SH approach performs well compared to the exact approach not only for $\zeta=0.4$, but also for $\zeta=0.0$. The reason for the latter value to work well is as follows. The system is initialized in the ground electronic state near the potential minimum. For trajectories that stay in this region, there is only a small coupling, and thus $\theta$ will remain around 0 in the optimization algorithm and the trajectories correctly evolve on the ground electronic state. Only if the trajectories have enough energy to escape from the potential minimum is there a significant coupling and the trajectories start to evolve on optimized, average 
$\mathrm{t}=10.00 \mathrm{fs}$
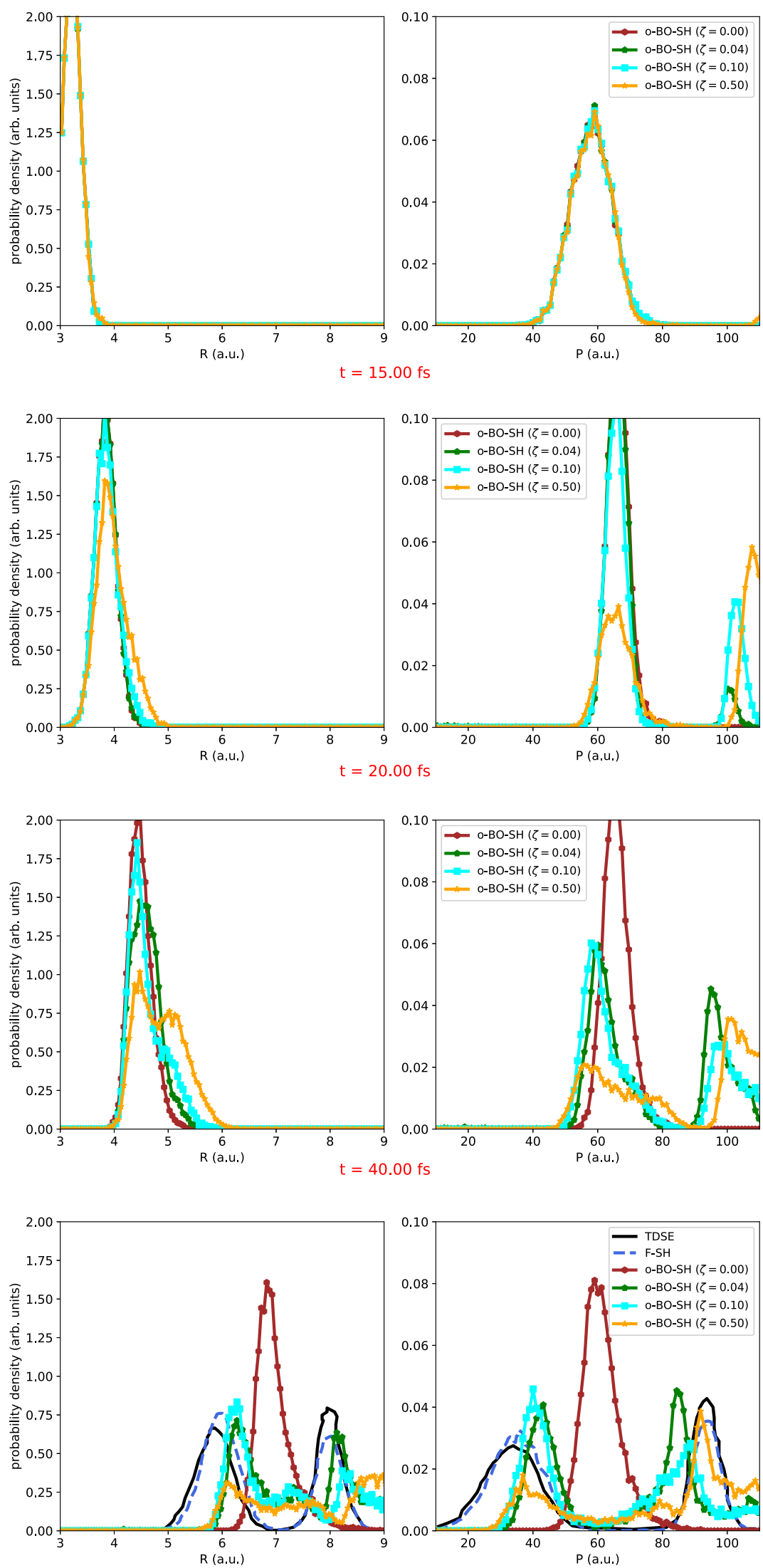

FIG. 4. Snapshots of nuclear densities at $t=10,15,20$, and $40 \mathrm{fs}$ in position (left panel) and momentum (right panel) space, o-BO-SH for $\zeta=0.5,0.1,0.04$, and 0.0. At $t=40 \mathrm{fs}$, the nuclear densities from o-BO-SH are compared to TDSE and F-SH from Ref. [57]. 

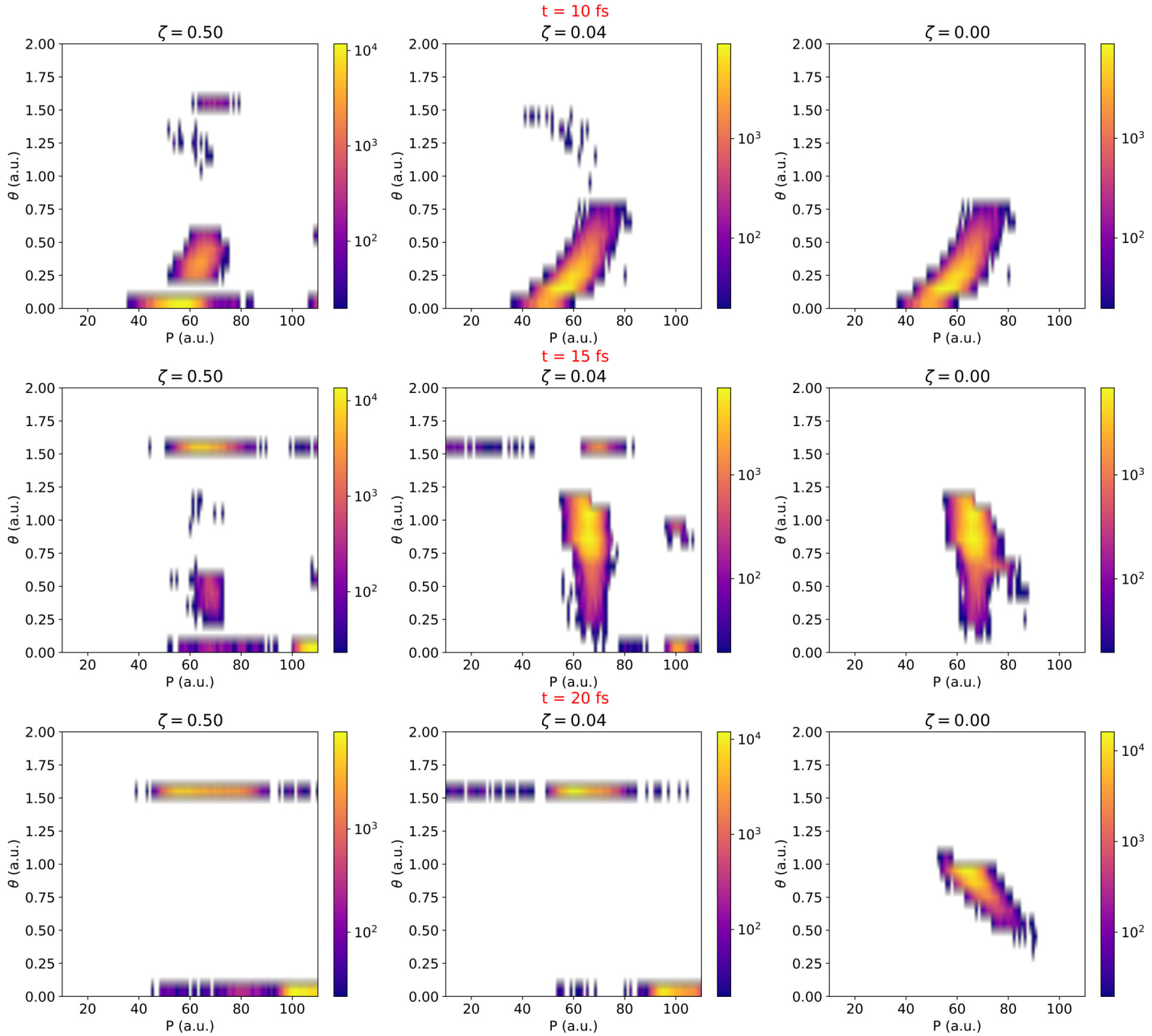

FIG. 5. The logarithmic distribution of momentum, $P$, and mixing parameter $\theta$, at $t=10,15$, and 20 fs employing o-BO-SH for $\zeta=0.50$ (left panels), 0.04 (center panels), and 0.0 (right panels).

states. However, for this dissociation case, there is no problem as the electronic states are degenerate in the dissociation limit.

Figure 7 shows the nuclear momentum density as a function of the optimization parameter $\theta$ at $t=25$ fs from o-BO$\mathrm{SH}$ for $\zeta=0.04$. Again, $\theta=0$ corresponds to a population of the lower BO PES, while a value of $\theta=\frac{\pi}{2}$ corresponds to a population of the upper BO PES. The two peaks in the momentum distribution can be directly associated with a population of the lower BO PES (peak around $P=0$ a.u.) and the second peak associated with a population of the upper BO PES (peak around $P=12$ a.u.), where the latter peak shows a broader $\theta$ distribution as both BO PESs are approximately degenerate at large distances.

\section{DISCUSSION}

For cases where the external field strongly couples the different BO PESs, it is best to describe each trajectory using a superposition of BO states, as done in the Ehrenfest approach, instead of a simple BO state, which is employed in FSSH. However, once the strong coupling is no longer present, the Ehrenfest-like approach breaks down as the system cannot be described with a coherent superposition any longer, but each state should be treated individually as is done in FSSH. The proposed method combines these in a straightforward and easy-to-implement way, which also gives accurate results.

Methods that follow a similar spirit as o-BO-SH have been proposed before [58-62], yet only for cases that do not include an external electric field. The continuous surface 


$$
\mathrm{t}=25 \mathrm{fs}
$$
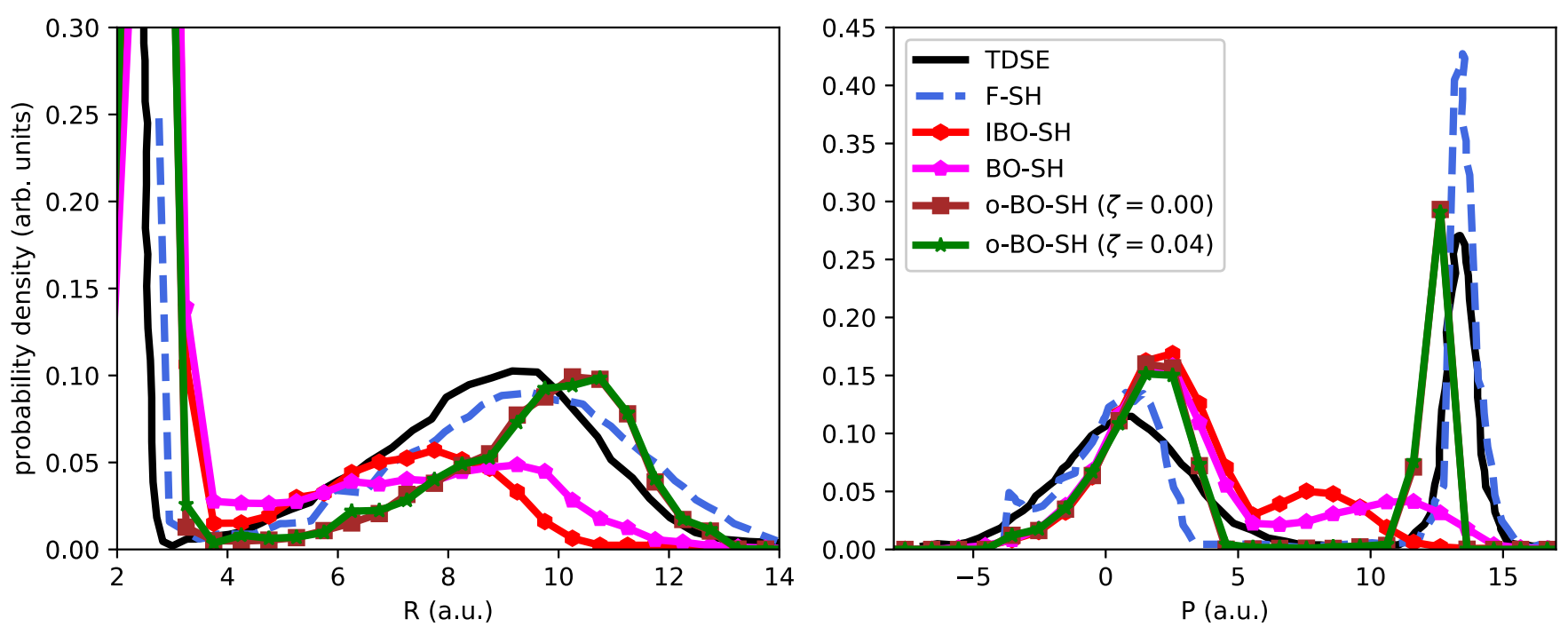

FIG. 6. The nuclear densities for scenario 2 at $t=25 \mathrm{fs}$, in position (left panel) and momentum (right panel) space, obtained from BO-SH, IBO-SH, o-BO-SH for $\zeta=0.00$ and o-BO-SH for $\zeta=0.04$, in the presence of a cw laser, in comparison to TDSE and F-SH from Ref. [57].

switching approaches (CSS, CCS2) [59,60] are variants of the Ehrenfest approach, but redefine the weights in the wave function. In areas of strong coupling, the weights should match the electronic populations and thus the approaches equal the Ehrenfest approach, while in areas of no coupling, the weights should be 0 or 1 to ensure correct dissociation. One of the important criteria for the definition of the weights is that the wave function should collapse in the regions of weak or no coupling. In that way, CSS and CSS2 are similar to the oBO-SH method, which also uses the current coupling strength as a criterion for the wave-function collapse. However, in our o-BO-SH, the collapse of the wave functions is instantaneous, while CSS and CSS2 have a continuous way of collapsing

$$
t=25 \mathrm{fs}
$$

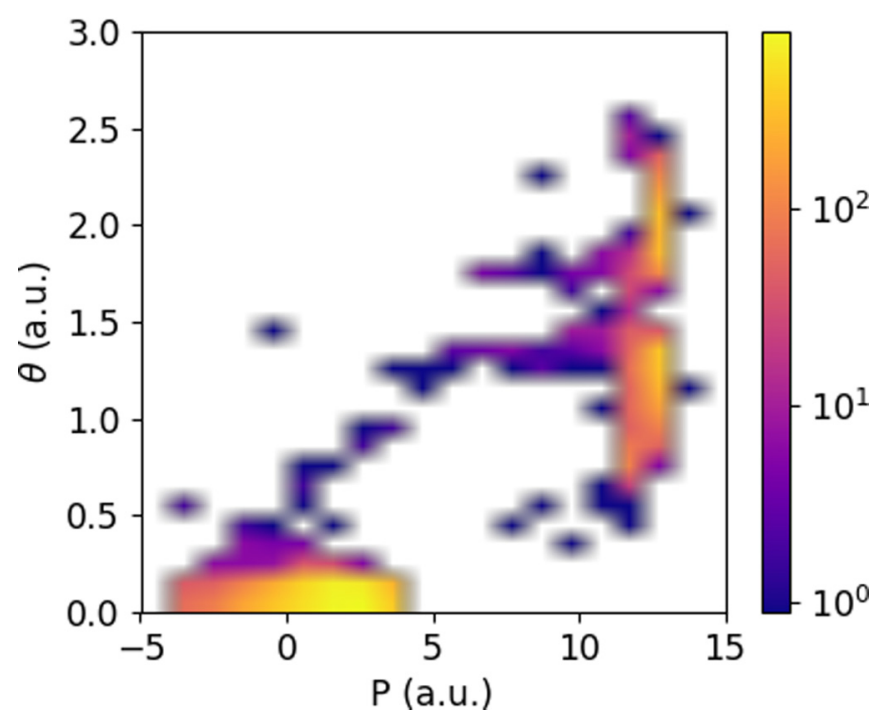

FIG. 7. The logarithmic distribution of momentum, $P$, and mixing parameter $\theta$, at $t=25 \mathrm{fs}$ employing o-BO-SH for $\zeta=0.04$. the wave function over some range of the potential. Meanfield dynamics with stochastic decoherence (MF-SD) [61] and augmented Ehrenfest (AE) [62] both have an instantaneous collapse of the wave function given by a rate. The rate is determined by analyzing either the difference between the Ehrenfest force and the forces for each electronic state [61] or by analyzing the Ehrenfest trajectories employing frozen Gaussians [62], which both differ from the o-BO-SH method that analyzes the coupling strength due to the external field.

For the two-state examples employed in this work, it was found that the optimization approach gives the same results as the Ehrenfest approach. It is not clear whether this holds true in any multistate case. Both approaches, i.e., the Ehrenfest approach and the optimization, can be easily implemented for a multistate case. The Ehrenfest approach is well known for any number of states, whereas for the optimization procedure, one needs to define a general, unitary matrix, perform the associated transformation of the electronic basis, and employ any standard optimization scheme to minimize the hopping probability.

\section{CONCLUSION}

For the mixed quantum classical description of the dynamics of molecules driven by coherent light fields, the FSH method from Ref. [57] overcomes certain limitations of the Ehrenfest, BO-SH, and IBO-SH methods. However, the Floquet method underlying F-SH imposes restrictions on the nature of the light pulses that can be treated. Particularly, few-cycle or subcycle pulses, which can be synthesized for applications in quantum control $[66,67]$, clearly violate the assumption of temporal (quasi-)periodicity inherent in the Floquet method. In contrast, the Ehrenfest, BO-SH, and IBO$\mathrm{SH}$ methods are not affected by this specific limitation of F-SH.

Therefore, in this work, we have explored another FSSH variant for computing field-induced dynamics in molecules. 
The proposed method combines an Ehrenfest-like approach for the strong-coupling region, which reduces the number of hops, and BO-SH in the weak-coupling regime. The switch between both approaches is performed based on a Rabioscillation model. The method is termed optimized BO-SH (o-BO-SH) and performs well compared to exact quantum results for different test cases of field-induced stimulated emission and photoabsorption employing a soft-core Hamiltonian. It is numerically efficient and can be easily generalized to treat multidimensional and multistate systems. It is left for future work to assess the accuracy of the method in these cases.

\section{ACKNOWLEDGMENTS}

We thank Oriol Vendrell for valuable discussions. This work was supported by the Cluster of Excellence "Advanced Imaging of Matter" of the Deutsche Forschungsgemeinschaft (DFG) - EXC 2056 - Project ID No. 390715994.
[1] A. Migliore, N. F. Polizzi, M. J. Therien, and D. N. Beratan, Chem. Rev. 114, 3381 (2014).

[2] M. Cordes and B. Giese, Chem. Soc. Rev. 38, 892 (2009).

[3] E. H. Jung, N. J. Jeon, E. Y. Park, C. S. Moon, T. J. Shin, T.-Y. Yang, J. H. Noh, and J. Seo, Nature (London) 567, 511 (2019).

[4] T. M. Brenner, D. A. Egger, L. Kronik, G. Hodes, and D. Cahen, Nat. Rev. Mater. 1, 15007 (2016).

[5] A. R. Arnold, M. A. Grodick, and J. K. Barton, Cell Chem. Biol. 23, 183 (2016).

[6] O. P. Ernst, D. T. Lodowski, M. Elstner, P. Hegemann, L. S. Brown, and H. Kandori, Chem. Rev. 114, 126 (2014).

[7] P. J. M. Johnson, A. Halpin, V. I. Prokhorenko, O. P. Ernst, and R. J. D. Miller, Nat. Chem. 7, 980 (2015).

[8] M. Barbatti, A. J. A. Aquino, J. J. Szymczak, D. Nachtigallová, P. Hobza, and H. Lischka, Proc. Natl. Acad. Sci. 107, 21453 (2010).

[9] K. Kleinermanns, D. Nachtigallová, and M. S. de Vries, Int. Rev. Phys. Chem. 32, 308 (2013).

[10] M. B. Smith and J. Michl, Annu. Rev. Phys. Chem. 64, 361 (2013).

[11] P. Ehrenfest, Z. Phys. 45, 455 (1927).

[12] O. V. Prezhdo and V. V. Kisil, Phys. Rev. A 56, 162 (1997).

[13] J. C. Tully, J. Chem. Phys. 93, 1061 (1990).

[14] J. C. Tully, Int. J. Quantum Chem. 40, 299 (1991).

[15] J. C. Tully, Faraday Discuss. 110, 407 (1998).

[16] P. V. Parandekar and J. C. Tully, J. Chem. Phys. 122, 094102 (2005).

[17] A. E. Sifain, L. Wang, and O. V. Prezhdo, J. Chem. Phys. 142, 224102 (2015).

[18] L. Wang, A. Akimov, and O. V. Prezhdo, J. Phys. Chem. Lett. 7, 2100 (2016).

[19] P. Shushkov, R. Li, and J. C. Tully, J. Chem. Phys. 137, 22 A549 (2012).

[20] R. Welsch, K. Song, Q. Shi, S. C. Althorpe, and T. F. Miller, J. Chem. Phys. 145, 204118 (2016).

[21] R. Kaur and R. Welsch, J. Chem. Phys. 150, 114105 (2019).

[22] F. A. Shakib and P. Huo, J. Phys. Chem. Lett. 8, 3073 (2017).

[23] X. Tao, P. Shushkov, and T. F. Miller, J. Chem. Phys. 148, 102327 (2018).

[24] X. Tao, P. Shushkov, and T. F. Miller, J. Phys. Chem. A 123, 3013 (2019).

[25] L. Wang, D. Trivedi, and O. V. Prezhdo, J. Chem. Theory Comput. 10, 3598 (2014).
[26] L. Wang, A. E. Sifain, and O. V. Prezhdo, J. Phys. Chem. Lett. 6, 3827 (2015).

[27] L. Wang, A. E. Sifain, and O. V. Prezhdo, J. Chem. Phys. 143, 191102 (2015).

[28] H. M. Jaeger, S. Fischer, and O. V. Prezhdo, J. Chem. Phys. 137, 22A545 (2012).

[29] A. V. Akimov and O. V. Prezhdo, Phys. Rev. Lett. 113, 153003 (2014).

[30] A. V. Akimov, D. Trivedi, L. Wang, and O. V. Prezhdo, J. Phys. Soc. Jpn. 84, 094002 (2015).

[31] L. Wang and O. V. Prezhdo, J. Phys. Chem. Lett. 5, 713 (2014).

[32] L. Wang and D. Beljonne, J. Phys. Chem. Lett. 4, 1888 (2013).

[33] A. V. Akimov, R. Long, and O. V. Prezhdo, J. Chem. Phys. 140, 194107 (2014).

[34] J. E. Subotnik and N. Shenvi, J. Chem. Phys. 134, 024105 (2011).

[35] V. N. Gorshkov, S. Tretiak, and D. Mozyrsky, Nat. Commun. 4, 2144 (2013).

[36] N. Shenvi, J. E. Subotnik, and W. Yang, J. Chem. Phys. 135, 024101 (2011).

[37] L. Wang, R. Long, and O. V. Prezhdo, Annu. Rev. Phys. Chem. 66, 549 (2015).

[38] A. V. Akimov and O. V. Prezhdo, Chem. Rev. 115, 5797 (2015).

[39] R. Mitrić, J. Petersen, and V. Bonačić Koutecký, Phys. Rev. A 79, 053416 (2009).

[40] T. Fiedlschuster, J. Handt, and R. Schmidt, Phys. Rev. A 93, 053409 (2016).

[41] Y. Suzuki, A. Abedi, N. T. Maitra, and E. K. U. Gross, Phys. Chem. Chem. Phys. 17, 29271 (2015).

[42] M. Richter, P. Marquetand, J. González-Vázquez, I. Sola, and L. González, J. Chem. Theory Comput. 7, 1253 (2011).

[43] I. Tavernelli, B. F. E. Curchod, and U. Rothlisberger, Phys. Rev. A 81, 052508 (2010).

[44] G. A. Jones, A. Acocella, and F. Zerbetto, J. Phys. Chem. A 112, 9650 (2008).

[45] R. Mitrić, J. Petersen, M. Wohlgemuth, U. Werner, V. BonačićKoutecký, L. Wöste, and J. Jortner, J. Phys. Chem. A 115, 3755 (2011)

[46] J. Petersen and R. Mitrić, Phys. Chem. Chem. Phys. 14, 8299 (2012).

[47] J. J. Bajo, G. Granucci, and M. Persico, J. Chem. Phys. 140, 044113 (2014).

[48] M. Fischer, J. Handt, and R. Schmidt, Phys. Rev. A 90, 012525 (2014). 
[49] M. Fischer, J. Handt, and R. Schmidt, Phys. Rev. A 90, 012526 (2014).

[50] M. Fischer, J. Handt, and R. Schmidt, Phys. Rev. A 90, 012527 (2014).

[51] F. Kelkensberg, G. Sansone, M. Y. Ivanov, and M. Vrakking, Phys. Chem. Chem. Phys. 13, 8647 (2011).

[52] J. J. Bajo, J. González-Vázquez, I. R. Sola, J. Santamaria, M. Richter, P. Marquetand, and L. González, J. Phys. Chem. A 116, 2800 (2012).

[53] I. Horenko, B. Schmidt, and C. Schütte, J. Chem. Phys. 115, 5733 (2001).

[54] P. Dietrich, M. Y. Ivanov, F. A. Ilkov, and P. B. Corkum, Phys. Rev. Lett. 77, 4150 (1996).

[55] M. Thachuk, M. Y. Ivanov, and D. M. Wardlaw, J. Chem. Phys. 105, 4094 (1996).

[56] M. Thachuk, M. Y. Ivanov, and D. M. Wardlaw, J. Chem. Phys. 109, 5747 (1998).

[57] T. Fiedlschuster, J. Handt, E. K. U. Gross, and R. Schmidt, Phys. Rev. A 95, 063424 (2017).
[58] M. Herman, J. Chem. Phys. 110, 4141 (1999).

[59] Y. L. Volobuev, M. D. Hack, M. S. Topaler, and D. G. Truhlar, J. Chem. Phys. 112, 9716 (2000).

[60] M. D. Hack and D. G. Truhlar, J. Chem. Phys. 114, 2894 (2001).

[61] M. J. Bedard-Hearn, R. E. Larsen, and B. J. Schwartz, J. Chem. Phys. 123, 234106 (2005).

[62] J. E. Subotnik, J. Chem. Phys. 132, 134112 (2010).

[63] M. K. Ganesa Subramanian, R. Santra, and R. Welsch, Phys. Rev. A 98, 063421 (2018).

[64] T. Tilma and E. C. G. Sudarshan, J. Phys. A 35, 10467 (2002).

[65] P. Virtanen et al., Nat. Methods 17, 261 (2020).

[66] A. Wirth, M. Th. Hassan, I. Grguras, J. Gagnon, A. Moulet, T. T. Luu, S. Pabst, R. Santra, Z. A. Alahmed, A. M. Azzeer, V. S. Yakovlev, V. Pervak, F. Krausz, and E. Goulielmakis, Science 334, 195 (2011).

[67] S.-W. Huang, G. Cirmi, J. Moses, K.-H. Hong, S. Bhardwaj, J. R. Birge, L.-J. Chen, E. Li, B. J. Eggleton, G. Cerullo, and F. X. Kärtner, Nat. Photon. 5, 475 (2011). 